\title{
ASCA X-RAY OBSERVATION OF THE LOBE DOMINANT RADIO GALAXY NGC 612
}

\author{
M. TASHIRO, H. KANEDA ${ }^{1}$, K. MAKISHIMA \\ Department of Physics, University of Tokyo \\ Hongo 7-3-1, Tokyo, Japan 113-0033
}

The radio galaxy NGC $612(z=0.0290$; Spinrad et al. 1985) exhibits bright ( $\sim 11 \mathrm{Jy}$ at $843 \mathrm{MHz}$ ) double lobes at a large scale of about $500 \mathrm{kpc}$ $\times 130 \mathrm{kpc}$ with a very faint core (Jones \& McAdam 1992). We performed $A S C A$ observations (AO-4; PI = Kaneda) of NGC 612 on July 12-14,1996, in search for inverse-Compton (IC) $\mathrm{X}$-rays from the radio lobes.

Figure 1 shows X-ray images of the central $\sim 10^{\prime}$ field around NGC 612 , obtained with the GISs in $0.7-3 \mathrm{keV}$ and 3-10 keV bands separately. The soft-band brightness map reveals an anisotropical diffuse emission in comparison with that in hard-band. The emission extends in the direction of the radio structure up to about $200 \mathrm{kpc}$ away from the core, exceeding typical spatial extent of diffuse X-ray emission observed from elliptical galaxies (Matsushita 1997). On the other hand, the point-like hard-band emission originates from the host galaxy. The X-ray spectrum obtained from the host galaxy region suggests heavily absorbed active nucleus.

We examined the X-ray spectrum of the extended emission and found it described with a power-law of photon index $1.8 \pm 0.4$. The agreement between the obtained spectral slope and that of the radio emission from the lobes strongly suggests that the $\mathrm{X}$-rays are produced via the IC process. This is the third detection of the IC X-rays from radio lobes, following the cases of Fornax A (Kaneda et al. 1995; Feigelson et al. 1995) and Centaurus B (PKS 1343-601: Tashiro et al. 1997). The estimated flux from NGC 612 lobes is $2.7 \times 10^{-13} \mathrm{erg} \mathrm{s}^{-1} \mathrm{~cm}^{-2}$ in the $0.5-10 \mathrm{keV}$ energy band. We derived the physical quantities to summarize in Table 1, according to Harris and Grindlay (1979). We also show derived quantities from Fornax $\mathrm{A}$ and Centaurus $\mathrm{B}$ for comparison. We conclude that the obtained results

\footnotetext{
${ }^{1}$ present address: Institute of Space and Astronautical Science Yoshinodai 3-1-1, Sagamihara, Japan 299-8510
} 

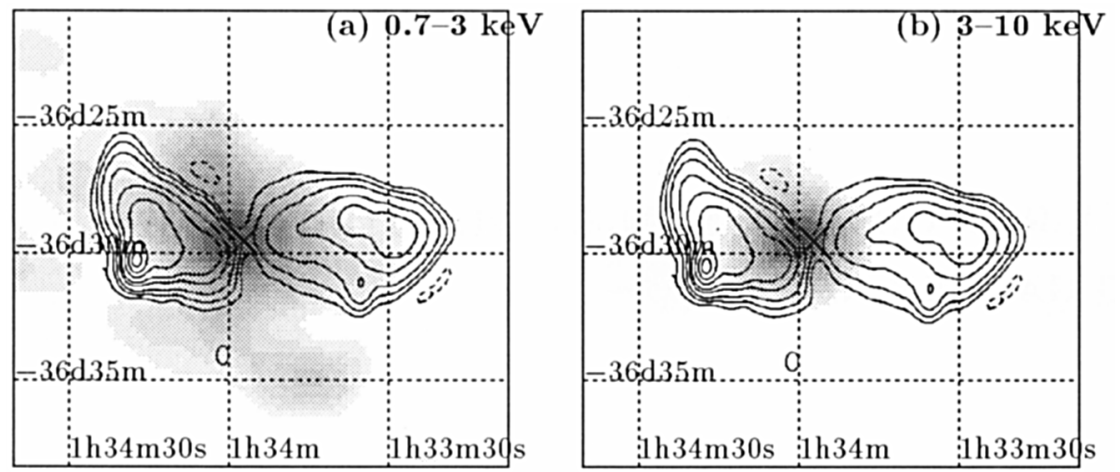

Figure 1. X-ray images of NGC 612 observed with the two GISs in (a) $0.7-3 \mathrm{keV}$ and (b) 3-10 keV. The non-X-ray background and the cosmic X-ray background have been subtracted using the night earth and blank sky data, respectively. The images have been vignetting corrected and smoothed with a Gaussian kernel of $\sigma=1^{\prime}$. Radio (843 $\mathrm{MHz}$ ) intensity contours from NGC 612 is superposed the X-ray images (Jones and McAdam 1992), and the cross is the optical core (Westerlund and Smith 1966).

from NGC 612 lobes are consistent with the energy equipartition hypothesis, although it prefers the particle dominant picture as Tashiro et al. (1997) showed in the case of Centaurus B.

TABLE 1. Evaluated Physical Quantities

\begin{tabular}{lccc}
\hline source & Fronax A & Centaurus B & NGC 612 \\
\hline magnetic energy density $\left[10^{-13} \mathrm{erg} \mathrm{cm}^{-3}\right]$ & $3.6 \pm 1.1$ & $3.8 \pm 1.8$ & $1.0 \pm 0.7$ \\
electron energy density $\left[10^{-13} \mathrm{erg} \mathrm{cm}^{-3}\right]$ & $3.0 \pm 1.3$ & $24 \pm 9$ & $2.6 \pm 1.7$ \\
\hline
\end{tabular}

\section{References}

Ekers, Goss, Kotanyi, \& Skellern, 1978, A\&A 69, L2

Harris, \& Grindlay, 1979, MNRAS 188, 25

Kaneda, et al. 1995, ApJ 453, L13

Jones, \& McAdam, 1992, ApJ SS 80, 137

Matsushita, 1997, PhD thesis, University of Tokyo

Spinrad, Djorgovski, Marr, \& Aguilar, 1985, Publ. Astron. Soc. Pac. 97.932

Tashiro, et al. 1997, ApJ submitted

Westerlund, B. E. \& Smith, L. F. 1966, Australian J. Phys. 19, 181 